\title{
Pneumococci responsible for invasive disease and discharging ears in children in Sydney, Australia
}

\author{
Michael Watson,† Maggie Brett, Mitchell Brown,‡ Marianne G. Stewart \\ and Shirley Warren, for the New South Wales Pneumococcal Network§
}

Correspondence

Michael Watson

mwatson@clinipath.net

\begin{abstract}
Received 8 November 2006
\end{abstract}
Accepted 1 February 2007

\begin{abstract}
The New South Wales Pneumococcal Reference Laboratory, The Children's Hospital at Westmead, Department of Microbiology, Westmead, New South Wales, Australia
\end{abstract}

\begin{abstract}
The serotypes and molecular clones of penicillin-nonsusceptible Streptococcus pneumoniae (PNSP) responsible for invasive pneumococcal disease (IPD) and discharging ears in metropolitan New South Wales were characterized to form a baseline prior to introduction of the heptavalent conjugate pneumococcal vaccine in Australia. Pneumococci isolated between 1 July 2000 and 30 June 2003 in Sydney from children $<15$ years were tested for antibiotic susceptibilities and serotyped. Penicillin-nonsusceptible pneumococci were typed by multilocus sequence typing and BOX PCR. During this period, 97 (13.9\%) of 698 pneumococci from IPD that were serotyped were penicillin-nonsusceptible. Of 607 pneumococci from discharging ears, 157 (26.1\%) were penicillin-nonsusceptible. Serotype 14 was the predominant serotype responsible for IPD and serotype 19F predominated from discharging ears. The heptavalent vaccine serotypes accounted for $613(87.8 \%)$ of all invasive isolates and $420(69.8 \%)$ of all isolates from discharging ears. Representatives of the major international clones were present among the PNSP. The majority of serotypes and clones that showed penicillin-nonsusceptibility are present within the vaccine. Serotype switching was also noted to have occurred prior to introduction of the vaccine. This study provides a characterization of the pneumococcal serotypes associated with IPD and discharging ears that will be useful for detecting potential selective effects of the vaccine. This surveillance should be continued, as it will be important to monitor the frequency and distribution of serotypes in the post-vaccine era.
\end{abstract}

\section{INTRODUCTION}

Streptococcus pneumoniae is a major cause of morbidity and mortality in children and adults in Australia and worldwide (Hogg et al., 2000; Jette \& Lamothe, 1989; Kertesz et al., 1998; Nielsen \& Henrichsen, 1992). Although penicillin resistance was first described in 1967 (Hansman \& Bullen, 1967), it did not become a significant problem in Australia until the 1990s (Turnidge et al., 1999). Trials with a heptavalent conjugate vaccine have shown it to be highly effective in the prevention of invasive pneumococcal disease (IPD) (Black \& Shinefield, 2002; Whitney et al., 2003); however, its efficacy for the prevention of middle ear infection is serotype-dependent (Eskola et al., 2001). The aim of this study was to examine the serotypes and

tPresent address: Clinipath Pathology, 647 Murray St, West Perth, Western Australia, 6005.

‡Present address: Institute of Clinical Pathology and Medical Research, Westmead Hospital, New South Wales, Australia.

§Members listed in Acknowledgements.

Abbreviations: IPD, invasive pneumococcal disease; MLST, multilocus sequence typing; PNSP, penicillin-nonsusceptible Streptococcus pneumoniae. molecular clones of penicillin-nonsusceptible S. pneumoniae (PNSP) responsible for IPD and discharging ears in metropolitan New South Wales (NSW) to form a baseline prior to introduction of the heptavalent conjugate pneumococcal vaccine in Australia. This is the first indepth description of the molecular clones of pneumococci responsible for penicillin-nonsusceptibility in Australia.

\section{METHODS}

Study population and data collection. The NSW Pneumococcal Network comprises all public and private microbiology laboratories in metropolitan NSW, processing all the clinical microbiology specimens for the Greater Sydney region, which has a population of approximately 5 million people. Isolates from patients fulfilling the case definitions were referred for susceptibility testing and molecular typing to the NSW Pneumococcal Reference Laboratory. IPD was defined as isolation of $S$. pneumoniae from a normally sterile body site. It did not include patients with suspected pneumococcal infection or those with only a positive pneumococcal antigen test. Discharging ear isolates included all isolates of $S$. pneumoniae isolated from ear swabs. No clinical data on discharging ear isolates were available. Isolates received between 1 July 2000 and 30 June 2003 from children less than 15 years were included in this study. 
Susceptibility testing. Susceptibility testing was performed using the NCCLS disc diffusion method on Mueller-Hinton agar with added sheep blood (NCCLS, 2003). Penicillin and cefotaxime MICs were evaluated using the E-test (AB Biodisk) on Mueller-Hinton agar with added sheep blood as per the manufacturer's recommendations. Pneumococci with penicillin MICs $\geqslant 0.12 \mathrm{mg}^{-1}$ were defined as PNSP and included isolates with intermediate (MICs $0.12-1 \mathrm{mg} \mathrm{l}^{-1}$ ) and high-level (MICs $\geqslant 2 \mathrm{mg} \mathrm{l}^{-1}$ ) penicillin resistance.

Serotyping. Isolates were serotyped by the Quellung reaction using rabbit polyclonal antisera from the Statens Serum Institute, Copenhagen, Denmark.

Molecular typing of PNSP. Molecular typing was performed using multilocus sequence typing (MLST) as described previously (Enright \& Spratt, 1998). Forty-two PNSP isolates (28 from invasive disease and 14 from ears) chosen to maximize a diverse range of serotypes, patient locations, age categories and resistance profiles were subjected to MLST and their sequence types were determined by comparison with sequences at the MLST website (www.mlst.net). Novel sequences of alleles and sequence types were submitted to the MLST website for confirmation. Representative isolates of each sequence type were then typed by BOX PCR fingerprinting as described previously (van Belkum et al., 1996) using the primers: BoxA, 5'-ATACTCTTCGAAAATCTCTTCAAAC-3'; BOXA1R, 5'-CTACGGCAAGGCGACGCTGACG- $3^{\prime}$. PCR products were run on a $2 \%$ ethidium bromidecontaining agarose gel in TBE for $1.5 \mathrm{~h}$ at $80 \mathrm{~V}$ and banding patterns were compared. BOX PCR fingerprinting was used to screen other PNSP isolates for assigning sequence types. PNSP isolates with BOX PCR profiles that could not be assigned to the sequence types determined by MLST were sequenced. For some sequence types, PCRRFLP of specific MLST alleles was also used in addition to BOX PCR fingerprinting. Sequence types ST236, ST320 and ST352 were differentiated by PCR-RFLP of the aroE allele with BsaHI, and AluI restriction digestion of the $d d l$ and $g k i$ alleles. Sequence types that shared five of seven alleles were considered to belong to a clonal complex and were compared with global clones identified by the Pneumococcal Molecular Epidemiology Network (PMEN) (www. sph.emory.edu/PMEN/).

\section{RESULTS AND DISCUSSION}

\section{Serotype and penicillin-nonsusceptibility in IPD}

During the study period there were 698 invasive isolates that were serotyped and had susceptibility results available. Of these, $97(13.9 \%)$ were penicillin-nonsusceptible. The serotypes of both penicillin-susceptible and -nonsusceptible isolates of $S$. pneumoniae responsible for IPD are displayed in Fig. 1. Serotype 14 was the predominant serotype responsible for IPD, accounting for $35.1 \%$ of all isolates. The next most common serotypes were $19 \mathrm{~F}$ $(13.1 \%), 6 \mathrm{~B}(12 \%)$ and $18 \mathrm{C}(8.9 \%)$. The heptavalent vaccine serotypes accounted for $613(87.8 \%)$ of all invasive isolates. Each serotype varied in its proportion of penicillin-nonsusceptibility, with serotypes $19 \mathrm{~F}$ and $9 \mathrm{~V}$ accounting for 30.9 and $26.8 \%$, respectively, of all the PNSP in IPD. The distribution of serotypes responsible for IPD in children in NSW is strikingly similar to the distribution of serotypes seen in the national Australian data for children less than 5 years (Watson et al., 2004). Serotype 14 was the predominant serotype responsible for IPD in this study and in the national Australian data. Similarly, serotype 14 was the predominant serotype in IPD in Scotland (Clarke et al., 2004) and in children in Portugal (Serrano et al., 2005).

\section{Serotype and penicillin-nonsusceptibility in ear isolates}

During the same time period, 607 discharging ear isolates were serotyped and had susceptibility results available. Of these, 157 (26.1\%) were penicillin-nonsusceptible. Among isolates from discharging ears (Fig. 2), serotype 19F was the predominant serotype $(207,34.4 \%)$. Other common

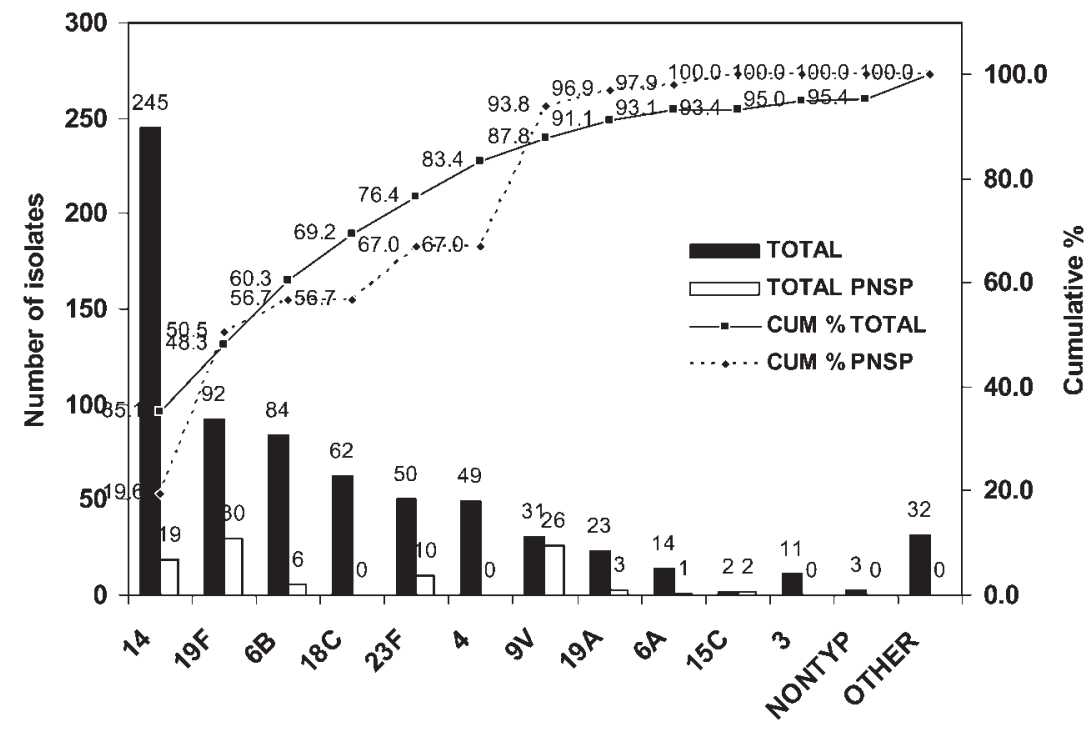

Pneumococcal serotype
Fig. 1. Pneumococcal serotypes and penicillinnonsusceptible S. pneumoniae in invasive pneumococcal disease in children in New South Wales, 1 July 2000-30 June 2003. 


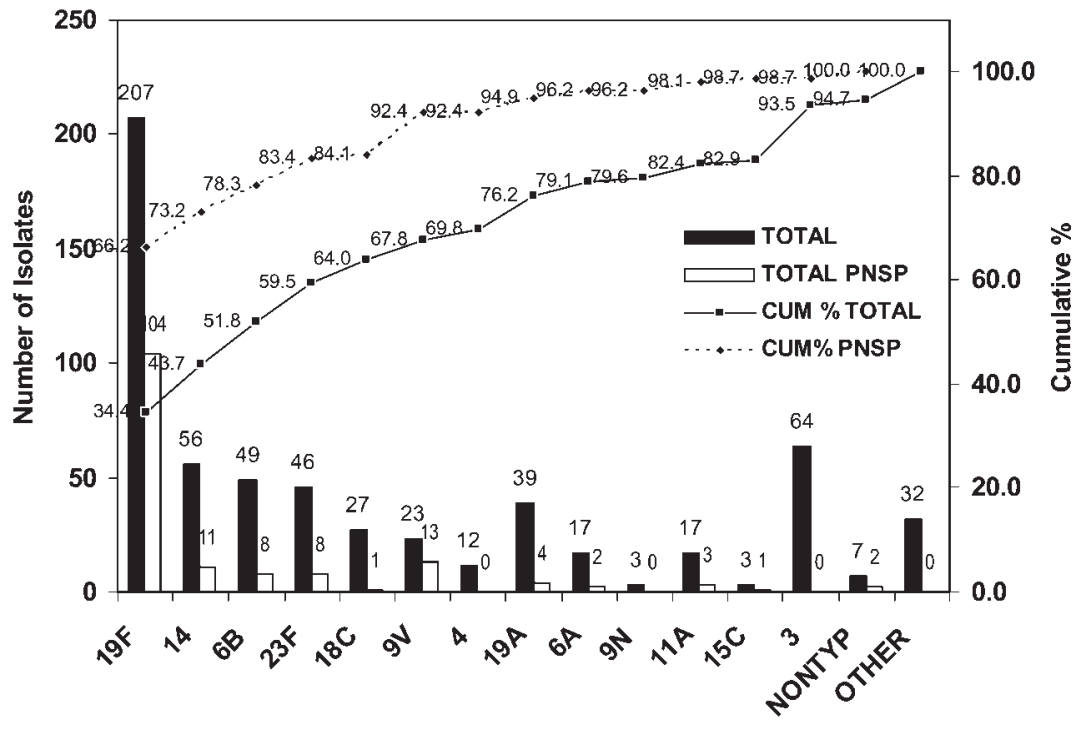

Pneumococcal serotype
Fig. 2. Pneumococcal serotypes and penicillinnonsusceptible $S$. pneumoniae in discharging ear isolates in children in New South Wales, 1 July 2000-30 June 2003. serotypes were serotype $3(10.6 \%), 14(9.3 \%), 6 \mathrm{~B}(8.1 \%)$ and $23 \mathrm{~F}(7.6 \%)$. Overall, heptavalent vaccine serotypes account for $420(69.8 \%)$ isolates. Of note is the relatively high proportion of penicillin-nonsusceptibility (104/207, $50.2 \%$ ) of serotype $19 \mathrm{~F}$, accounting for $66.2 \%$ of all PNSP from ears.

\section{Molecular clones of PNSP}

A total of 197 PNSP isolates were typed by MLST or BOX PCR fingerprinting and tested for susceptibilities to a range of antibiotics (Table 1). The distributions of molecular types were similar in PNSP from IPD and ear discharge. Among serotype 19F pneumococci, the majority (94.5\%) belonged to ST320, ST352, ST236, ST651 or ST257, all related to the PMEN international clone Taiwan ${ }^{19 F}-14$. Two of the sequence types (ST320 and ST352) were novel STs. ST320 was the predominant serotype $19 \mathrm{~F}$ clone among both the IPD and ear isolates and is a double locus variant of the Taiwan ${ }^{19 F}-14$ clone. All the serotype 9V PNSP belonged to ST156, the Spain ${ }^{9 \mathrm{~V}}-3$ clone. Of the 25 serotype 14 PNSP, 16 belonged to the Spain ${ }^{9 \mathrm{~V}}-3$ clonal complex, demonstrating serotype switching, and nine to the England $^{14}-9$ clonal complex. Among the serotype 23F PNSP, six were ST81 (Spain ${ }^{23 \mathrm{~F}}-1$ clone) and four belonged to the Taiwan ${ }^{23 \mathrm{~F}}-15$ clonal complex. Representatives (ST90) of the Spain ${ }^{6 \mathrm{~B}}-2$ clone predominated among the serotype $6 \mathrm{~B}$ PNSP.

Significant heterogeneity in PNSP strains occurred with multiple clones within multiple serotypes. However, representatives of two international clonal complexes, the Taiwan $^{19 \mathrm{~F}}-14$ and Spain ${ }^{9 \mathrm{~V}}-3$, accounted for the majority of PNSP (157/197, 79.6\%) from IPD and discharging ears. Other international PMEN clones found in this study were representatives of ST81, the Spain ${ }^{23 \mathrm{~F}}-1$ clone, ST90, the Spain $^{6 \mathrm{~B}}-2$ clone, and ST9, the England ${ }^{14}-9$ clone.
The majority of serotypes and clones that were penicillinnonsusceptible are present within the heptavalent vaccine. Most of the PNSP of serotypes 14 and 9V were representatives of ST156, the Spain ${ }^{9 \mathrm{~V}}-3$ clone. This clone has been shown to comprise the majority of the isolates of serotype $9 \mathrm{~V}$ in a study of invasive pneumococcal isolates in the USA (Gertz et al., 2003) and of serotypes $9 \mathrm{~V}$ and 14 in a similar study in Portugal (Serrano et al., 2005).

In our study, serotype 19F predominated from discharging ears. Of concern is the high rate of penicillin-nonsusceptibility in serotype $19 \mathrm{~F}$ and the relatively poor efficacy that this serotype has demonstrated in a clinical trial of the heptavalent conjugate vaccine in preventing otitis media (Eskola et al., 2001). Our data showed that the majority of penicillin-nonsusceptible serotype $19 \mathrm{~F}$ isolates belonged to ST320, a novel sequence type that is related to the Taiwan $^{19 F}$-14 clone. This clone is multi-resistant and has been isolated in Asia (Shi et al., 1998), Africa (McGee et al., 2001), the USA (Gertz et al., 2003) and Europe (www.mlst.net). Ongoing surveillance is essential to ensure that a reduction in ear disease due to this serotype will occur following the introduction of this vaccine into Australia. Notably, serotype 3 isolates accounted for $10 \%$ of the ear isolates. This serotype is not covered by the heptavalent vaccine.

This study provides a characterization of the pneumococcal serotypes associated with IPD and discharging ears that will be useful for detecting potential selective effects of the vaccine. Recent reports have shown an increase in the isolation of serotype 19A from IPD in children in the USA in the post-vaccine period (Pai et al., 2005). Thus surveillance should be continued, as it will be important to monitor the frequency and distribution of serotypes in the post-vaccine era. 
Table 1. Molecular clones of penicillin-nonsusceptible Streptococcus pneumoniae in New South Wales, Australia: 1 July $2000-30$ June 2003

Pen, Penicillin; Ctx, cefotaxime; Ery, erythromycin; Cli, clindamycin; Chl, chloramphenicol; Sxt, trimethoprim-sulfamethoxazole; Tet, tetracycline; NA, not applicable.

\begin{tabular}{|c|c|c|c|c|c|c|c|c|c|}
\hline \multirow[t]{2}{*}{ Serotype, $\mathrm{ST}^{\star}$} & \multirow[t]{2}{*}{ Clonal complex } & \multirow[t]{2}{*}{ No. tested } & \multicolumn{2}{|c|}{ Median MIC (range) $\mathrm{mg} \mathrm{l}^{-1}$} & \multicolumn{5}{|c|}{$\%$ Resistant (\% intermediate) } \\
\hline & & & Pen & Ctx & Ery & Cli & Chl & Sxt & Tet \\
\hline \multicolumn{10}{|l|}{$19 \mathrm{~F}$} \\
\hline ST $320 \dagger$ & Taiwan $^{19 \mathrm{~F}}-14$ & 65 & $2.0(1.5-6.0)$ & $1.5(0.75-3.0)$ & 100 & $94.5(1.5)$ & 0 & 100 & 97 \\
\hline ST $352 \dagger$ & Taiwan $^{19 F}-14$ & 30 & $1.0(0.5-3.0)$ & $0.75(0.5-1.5)$ & 100 & 100 & 0 & 100 & 100 \\
\hline ST 236 & Taiwan $^{19 \mathrm{~F}}-14$ & 8 & $1(0.5-1.0)$ & $0.5(0.25-0.75)$ & $87.5(12.5)$ & 12.5 & 0 & 75 & 100 \\
\hline ST 651 & Taiwan $^{19 \mathrm{~F}}-14$ & 1 & 0.75 & 0.38 & $0(100)$ & 0 & 0 & 100 & 100 \\
\hline ST 257 & Taiwan $^{19 \mathrm{~F}}-14$ & 1 & 0.19 & 0.19 & 100 & 100 & 0 & $0(100)$ & 100 \\
\hline ST 325 & - & 3 & $0.5(0.38-1.0)$ & $0.125(0.125-0.25)$ & 0 & 0 & 0 & $67(33)$ & 0 \\
\hline ST $1061 \dagger$ & Portugal $^{19 \mathrm{~F}}-21$ & 2 & $\begin{array}{l}0.125 \\
\quad(0.125-0.125)\end{array}$ & NA $(0.032-0.125)$ & 0 & 0 & 0 & 0 & 0 \\
\hline ST 33 & Tennessee $^{23 \mathrm{~F}}-4$ & 1 & 2 & 1.5 & 100 & 100 & 0 & 100 & 100 \\
\hline \multicolumn{10}{|l|}{14} \\
\hline ST 156 & Spain $^{9 V}-3$ & 14 & $1.5(0.75-2.0)$ & $0.75(0.25-1.0)$ & 21 & 21 & 0 & 93 & 21 \\
\hline ST 143 & Spain $^{9 \mathrm{~V}}-3$ & 2 & NA $(1.0-1.5)$ & $0.75(0.75-0.75)$ & 100 & 100 & 0 & 100 & 100 \\
\hline ST 9 & England $^{14}-9$ & 5 & $1.0(0.75-1.5)$ & $0.5(0.5-1.0)$ & 100 & 100 & 0 & 100 & 0 \\
\hline ST 29 & England $^{14}-9$ & 4 & $0.19(0.19-1.0)$ & $0.125(0.125-0.5)$ & $0(25)$ & 0 & 0 & 50 & 0 \\
\hline \multicolumn{10}{|l|}{$9 \mathrm{~V}$} \\
\hline ST 156 & Spain $^{9 V}-3$ & 36 & $1.5(0.75-2.0)$ & $0.75(0.38-1.5)$ & 6 & 3 & 0 & 100 & $3(3)$ \\
\hline \multicolumn{10}{|l|}{$23 \mathrm{~F}$} \\
\hline ST 81 & Spain $^{23 \mathrm{~F}}-1$ & 6 & $2.0(1.5-2.0)$ & $1.0(0.75-1.5)$ & 100 & 33 & 83 & 83 & 83 \\
\hline ST 880 & Taiwan $^{23 \mathrm{~F}}-15$ & 2 & NA $(1.0-2.0)$ & NA $(0.5-1.0)$ & 100 & 100 & 0 & 100 & 100 \\
\hline ST 242 & Taiwan $^{23 \mathrm{~F}}-15$ & 2 & $2.0(2.0-2.0)$ & NA $(1.0-1.5)$ & 100 & 0 & 0 & $50(50)$ & 100 \\
\hline $\begin{array}{l}\text { ST } 172 \\
\text { 6B }\end{array}$ & Columbia $^{23 \mathrm{~F}}-26$ & 1 & 0.75 & 0.75 & 0 & 0 & 0 & 100 & 0 \\
\hline ST 90 & Spain $^{6 \mathrm{~B}}-2$ & 7 & $1.0(0.75-1.0)$ & $0.5(0.5-0.75)$ & 100 & 100 & 86 & 100 & 100 \\
\hline ST 315 & Poland ${ }^{6 \mathrm{~B}}-20$ & 1 & 0.094 & 0.032 & 100 & 100 & 0 & 0 & 100 \\
\hline ST 185 & S. Africa ${ }^{6 \mathrm{~B}}-8$ & 2 & $0.25(0.25-0.25)$ & $0.19(0.19-0.19)$ & 0 & 0 & 0 & 100 & 0 \\
\hline $\begin{array}{l}\text { ST } 1056 \dagger \\
\text { 15C }\end{array}$ & S. Africa ${ }^{6 \mathrm{~B}}-8$ & 1 & 0.125 & 0.125 & 0 & 0 & 0 & 100 & 0 \\
\hline ST 346 & - & 2 & $0.38(0.38-0.38)$ & NA $(0.094-0.19)$ & 0 & 0 & 0 & 0 & 0 \\
\hline ST $857 \dagger$ & - & 1 & 0.38 & 0.25 & 0 & 0 & 0 & 0 & 0 \\
\hline
\end{tabular}

${ }^{\star}$ Sequence type determined by MLST or BOX PCR.

$\dagger$ Sequence types first isolated in this study.

\section{ACKNOWLEDGEMENTS}

We acknowledge the use of the pneumococcal MLST database, located at Imperial College London and funded by the Wellcome Trust.

The New South Wales Pneumococcal Network: Central Coast Pathology, Mr Bruce Beaman and Dr Deo Dewitt; Concord Hospital, Ms Candice Wolfson and Dr Tom Gottlieb; Davies Campbell \& De Lambert Pathology, Mr Steve Hodges and Dr De Lambert; Douglass Hanley Moir Pathology, Mr Richard Jones and Dr Ian Chambers; Hunter Area Pathology Service, Mr Chris AshurstSmith and Dr John Ferguson; CIDM-ICPMR, Mr David Smith and Professor G. L. Gilbert; Laverty Pathology, Mr David Rankin and Dr Juliette Holland; NCIRS, Professor Peter McIntyre and Ms Robin Gilmour; THE Pathology, Mr Andrew Jarrett and Dr Val Ackerman; Nepean Hospital, Mr David Rose and Dr James Branley; PaLMS Pathology, Dr Clarence Fernandes and Dr Robert Pritchard; Royal Prince Alfred Hospital, Ms Barbara Yan and Professor Richard Benn;
SEALS Pathology, Ms Sue Mahrer and A/Professor John Tapsall; St George Hospital, Ms Kerry Varettas and Dr Peter Taylor; St Vincents Hospital, Mr Damien Stark and Dr Jock Harkness; SWAPS Pathology, Mr Steven Neville and Dr Iain Gosbell; Sydney Adventist Hospital, Dr Ross Grant and Dr Ross Bradbury; IAHS Pathology, Mr David Andriske and Dr Peter Newton.

\section{REFERENCES}

Black, S. \& Shinefield, H. R. (2002). Safety and efficacy of the sevenvalent pneumococcal conjugate vaccine: evidence from Northern California. Eur J Pediatr 161 (Suppl. 2), S127-S132.

Clarke, S. C., Scott, K. J. \& McChlery, S. M. (2004). Serotypes and sequence types of pneumococci causing invasive disease in Scotland prior to the introduction of pneumococcal conjugate polysaccharide vaccines. J Clin Microbiol 42, 4449-4452. 
Enright, M. C. \& Spratt, B. G. (1998). A multilocus sequence typing scheme for Streptococcus pneumoniae: identification of clones associated with serious invasive disease. Microbiology 144, 3049-3060.

Eskola, J., Kilpi, T., Palmu, A., Jokinen, J., Haapakoski, J., Herva, E., Takala, A., Kayhty, H., Karma, P. \& other authors (2001). Efficacy of a pneumococcal conjugate vaccine against acute otitis media. Finnish Otitis Media Study Group. N Engl J Med 344, 403-409.

Gertz, R. E., McEllistrem, M. C., Boxrud, D. J., Li, Z., Sakota, V., Thompson, T. A., Facklam, R. R., Besser, J. M., Harrison, L. H. \& other authors (2003). Clonal distribution of invasive pneumococcal isolates from children and selected adults in the United States prior to 7-valent conjugate vaccine introduction. J Clin Microbiol 41, 4194-4216.

Hansman, D. \& Bullen, M. M. (1967). A resistant pneumococcus. Lancet 2, 264-265.

Hogg, G. G., Strachan, J. E. \& Lester, R. A. (2000). Invasive pneumococcal disease in the population of Victoria. Med J Aust 173 (Suppl.), S32-S35.

Jette, L. P. \& Lamothe, F. (1989). Surveillance of invasive Streptococcus pneumoniae infection in Quebec, Canada, from 1984 to 1986: serotype distribution, antimicrobial susceptibility, and clinical characteristics. J Clin Microbiol 27, 1-5.

Kertesz, D. A., Di Fabio, J. L., de Cunto Brandileone, M. C., Castaneda, E., Echaniz-Aviles, G., Heitmann, I., Homma, A., Hortal, M., Lovgren, M. \& other authors (1998). Invasive Streptococcus pneumoniae infection in Latin American children: results of the Pan American Health Organization Surveillance Study. PAHO Pneumococcal Surveillance Study Group. Clin Infect Dis 26, 1355-1361.

McGee, L., Klugman, K. P., Wasas, A., Capper, T. \& Brink, A. Antibiotics Surveillance Forum of South Africa (2001). Serotype 19F multiresistant pneumococcal clone harbouring two erythromycin resistance determinants [erm(B) and mef(A)] in South Africa. Antimicrob Agents Chemother 45, 1595-1598.
NCCLS (2003). Performance Standards for Antimicrobial Disk Susceptibility Test, 8th edn. Approved standard M2-A8. Wayne, PA: National Committee for Laboratory Standards.

Nielsen, S. V. \& Henrichsen, J. (1992). Capsular types of Streptococcus pneumoniae isolated from blood and CSF during 1982-1987. Clin Infect Dis 15, 794-798.

Pai, R., Moore, M. R., Pilishvili, T., Gertz, R. E., Whitney, C. G. \& Beall, B. Active Bacterial Core Surveillance Team (2005). Postvaccine genetic structure of Streptococcus pneumoniae serotype 19A from children in the United States. J Infect Dis 192, 1988-1995.

Serrano, I., Melo-Cristino, J., Carriço, J. A. \& Ramirez, M. (2005). Characterization of the genetic lineages responsible for pneumococcal invasive disease in Portugal. J Clin Microbiol 43, 1706-1715.

Shi, Z. Y., Enright, M. C., Wilkinson, P., Griffiths, D. \& Spratt, B. G. (1998). Identification of the three major clones of multiply antibioticresistant Streptococcus pneumoniae in Taiwanese hospitals by multilocus sequencing typing. J Clin Microbiol 36, 3514-3519.

Turnidge, J. D., Bell, J. M. \& Collignon, P. J. (1999). Rapidly emerging antimicrobial resistances in Streptococcus pneumoniae in Australia. Med J Aust 170, 152-155.

van Belkum, A., Sluijter, M., de Groot, R., Verbrugh, H. \& Hermans, P. W. M. (1996). Novel BOX repeat PCR assay for high-resolution typing of Streptococcus pneumoniae strains. J Clin Microbiol 34, 1176-1179.

Watson, M., Roche, P., Bayley, K., Bell, J. M., Collignon, P., Gilbert, G. L., Hogg, G., Keil, A. D., Krause, V. \& other authors (2004). Laboratory surveillance of invasive pneumococcal disease in Australia, 2003 predicting the future impact of the universal childhood conjugate vaccine program. Commun Dis Intell 28, 455-464.

Whitney, C. G., Farley, M. M., Hadler, J., Harrison, L. H., Bennett, N. M., Lynfield, R., Reingold, A., Cieslak, P. R., Pilishvili, T. \& other authors (2003). Decline in invasive pneumococcal disease after the introduction of protein-polysaccharide conjugate vaccine. Active Bacterial Core Surveillance of the Emerging Infections Program Network. $N$ Engl J Med 348, 1737-1746. 